\title{
Hipparcos Observations of Hydrogen-deficient Carbon Stars*
}

\author{
P. L. Cottrell ${ }^{1}$ and W. A. Lawson ${ }^{2}$ \\ ${ }^{1}$ Mount John University Observatory, Department of Physics and Astronomy, \\ University of Canterbury, Christchurch, New Zealand \\ p.cottrell@phys.canterbury.ac.nz \\ ${ }^{2}$ School of Physics, University College, UNSW, Australian Defence Force Academy, \\ Canberra, ACT 2600, Australia \\ w-lawson@adfa.oz.au \\ Received 1997 August 1, accepted 1997 December 1
}

\begin{abstract}
Parallax measurements for 21 hydrogen-deficient carbon stars have been made by the Hipparcos satellite. These stars include most of the brighter $\mathrm{R}$ Coronae Borealis (RCB) variables, other cool hydrogen-deficient carbon (HdC) stars, and several higher- $T_{\text {eff }}$ extreme helium (eHe) stars. Most of these stars have either negative or statistically insignificant parallaxes, indicating that they lie beyond the detection capability of Hipparcos. Although the distances to the galactic hydrogen-deficient carbon stars remain unknown, at least the Hipparcos observations do confirm that these objects must have high luminosity like the LMC RCB stars, for which $M_{\text {bol }}=$ -4 to -5 . Based upon Hipparcos proper motions, we derive $U V W$ velocities for the $\mathrm{RCB}$ and $\mathrm{HdC}$ stars, assuming $M_{\mathrm{bol}}=-3$ and -5 . The $U W$-velocity dispersion of the $\mathrm{RCB} / \mathrm{HdC}$ stars is similar to that already reported for the eHe stars, further supporting the idea that these groups of stars have predominantly bulge distributions. However, UW Cen may be a second example of a halo RCB star currently seen transitting the Galactic plane.
\end{abstract}

Keywords: stars: AGB and post-AGB — stars: carbon — stars: distances stars: fundamental parameters — stars: kinematics

\section{Introduction}

The hydrogen-deficient carbon (HdC) stars consist of several groups of probably related objects with a combined known population in the Galaxy of about 100 stars. They are believed to be examples of postAGB objects. Most exhibit a high degree of hydrogen depletion (up to factors of $10^{5}$ ), although their metal abundances range from $0 \cdot 1$ solar to solar (Lambert \& Rao 1994). The cooler hydrogen-deficient stars are generally separated into (1) the RCB stars, highly variable objects well known for their largeamplitude dust-formation events (declines) (Cottrell 1996; Clayton 1996) and (2) the HdC stars, which may be low-activity RCB stars on account of their lower pulsational activity (Lawson \& Cottrell 1997) and evidence for limited mass loss (Walker 1986). The distinction between these two groups is often arbitrary, e.g. XX Cam has been variously classified as $\mathrm{RCB}$ or HdC, depending upon different characteristics of the star. The eHe stars are distinguished from the cool $\mathrm{RCB} / \mathrm{HdC}$ stars in terms of $T_{\text {eff }}$; the cool $\mathrm{RCB} / \mathrm{HdC}$ stars have $T_{\text {eff }} \approx 5000-7500 \mathrm{~K}$, whereas the eHe stars have $T_{\text {eff }}$ exceeding $\sim 8000 \mathrm{~K}$.

\footnotetext{
* Based on data from the ESA Hipparcos astrometry satellite.

(C) Astronomical Society of Australia 1998
}

Spectroscopic analyses of these stars (Cottrell \& Lambert 1982; Pollard, Cottrell \& Lawson 1994; Lambert \& Rao 1994) indicate that these are lowgravity objects $(\log g \approx 0-1)$. The only distance estimates, and therefore observationally determined values for $M_{V}$ or $M_{\mathrm{bol}}$, are for RCB stars in the LMC.

Three LMC RCB stars have been known since their discovery on the Harvard Observatory survey plates during the early decades of this century, but a larger population is currently emerging from the MACHO inventory of variable stars in the LMC. W Men and HV 12842 are F-type RCB stars, spectroscopically similar to the prototype Galactic $\mathrm{RCB} / \mathrm{HdC}$ stars R CrB and RY Sgr (Pollard et al. 1994). Assuming a distance modulus to the LMC of $18 \cdot 7$ (Feast \& Catchpole 1997), W Men and HV 12842 have $M_{V}$ of $-4 \cdot 9$ and $-5 \cdot 0$ at maximum light, respectively. The cooler (K spectral type) LMC RCB star HV 5637 (Lawson et al. 1990; Glass, Lawson \& Laney 1994) has $M_{V}=-4 \cdot 2$. Early results from the MACHO survey (Alcock et al. 1996; Clayton 1998) suggest a larger population (10-20 confirmed or probable RCB stars in fields examined to date) dominated by cooler, less-luminous stars with $M_{V}$ $=-3$ to -4 . After bolometric corrections are accounted for, these objects have mean luminosities

1323-3580/98/020179\$05.00 
Table 1. Hipparcos data for 21 hydrogen-deficient carbon stars

\begin{tabular}{|c|c|c|c|c|c|c|c|c|c|c|}
\hline Star & Type & $\begin{array}{c}\text { Number } \\
\text { (HIP) }\end{array}$ & \multicolumn{2}{|c|}{$(\mathrm{J} 1991 \cdot 25)$} & $\begin{array}{c}\pi \\
(\mathrm{mas})\end{array}$ & \multicolumn{2}{|c|}{$\begin{array}{c}\mu_{\alpha} \\
\left(\operatorname{mas~yr}^{-1}\right)\end{array}$} & $\begin{array}{c}\sigma \pi \\
(\operatorname{mas})\end{array}$ & $\begin{array}{c}\sigma \mu_{\alpha} \\
(\mathrm{mas}\end{array}$ & $\begin{array}{c}\sigma \mu_{\delta} \\
\left.\mathrm{r}^{-1}\right)\end{array}$ \\
\hline XX Cam & $\mathrm{HdC}$ & 019340 & $040838 \cdot 75$ & $+532139 \cdot 4$ & $-1 \cdot 04$ & $1 \cdot 09$ & $-3 \cdot 46$ & $0 \cdot 90$ & $0 \cdot 74$ & $0 \cdot 71$ \\
\hline SU Tau & $\mathrm{RCB}$ & 027465 & $054858 \cdot 68$ & $+190436 \cdot 4$ & $3 \cdot 32$ & $3 \cdot 57$ & $1 \cdot 17$ & $5 \cdot 24$ & $6 \cdot 16$ & $3 \cdot 28$ \\
\hline $\mathrm{BD}+10^{\circ} 2179$ & $\mathrm{eHe}$ & 052133 & $103855 \cdot 24$ & $+100348 \cdot 5$ & $0 \cdot 49$ & $-12 \cdot 05$ & $-3 \cdot 18$ & $1 \cdot 61$ & $1 \cdot 83$ & $1 \cdot 55$ \\
\hline UW Cen & $\mathrm{RCB}$ & 062071 & $124317 \cdot 20$ & $-543140 \cdot 7$ & $1 \cdot 27$ & $-7 \cdot 87$ & $-12 \cdot 64$ & $2 \cdot 81$ & $3 \cdot 26$ & $3 \cdot 89$ \\
\hline Y Mus & $\mathrm{RCB}$ & 063911 & $130548 \cdot 20$ & $-653046 \cdot 6$ & $8 \cdot 02$ & $-4 \cdot 14$ & $0 \cdot 08$ & $1 \cdot 93$ & $1 \cdot 60$ & $1 \cdot 70$ \\
\hline HD 124448 & $\mathrm{eHe}$ & 069619 & $141458 \cdot 64$ & $-4617 \quad 19 \cdot 3$ & 0.59 & $-7 \cdot 54$ & $1 \cdot 01$ & $1 \cdot 75$ & $1 \cdot 20$ & $1 \cdot 30$ \\
\hline S Aps & $\mathrm{RCB}$ & 074179 & $150924 \cdot 55$ & $-720345 \cdot 2$ & $-0 \cdot 89$ & $-5 \cdot 45$ & $-0 \cdot 76$ & $1 \cdot 56$ & $1 \cdot 37$ & $1 \cdot 68$ \\
\hline HD 137613 & $\mathrm{HdC}$ & 075694 & $152748 \cdot 32$ & $-251010 \cdot 1$ & $-1 \cdot 36$ & $-1 \cdot 84$ & $-8 \cdot 11$ & $1 \cdot 32$ & $1 \cdot 29$ & $0 \cdot 94$ \\
\hline $\mathrm{R} \mathrm{CrB}$ & $\mathrm{RCB}$ & 077442 & $154834 \cdot 42$ & $+280924 \cdot 4$ & 0.54 & $-2 \cdot 10$ & $-11 \cdot 52$ & $0 \cdot 72$ & $0 \cdot 37$ & $0 \cdot 49$ \\
\hline HD 148839 & $\mathrm{HdC}$ & 081254 & $163545 \cdot 80$ & $-670736 \cdot 7$ & $1 \cdot 47$ & $-5 \cdot 31$ & $-3 \cdot 20$ & $1 \cdot 11$ & $0 \cdot 72$ & $0 \cdot 88$ \\
\hline HD 160641 & $\mathrm{eHe}$ & 086605 & $174151 \cdot 58$ & $-175348 \cdot 5$ & $-1 \cdot 20$ & $-1 \cdot 64$ & $1 \cdot 57$ & $2 \cdot 14$ & $2 \cdot 28$ & $1 \cdot 55$ \\
\hline RS Tel & $\mathrm{RCB}$ & 089739 & $181851 \cdot 22$ & $-463253 \cdot 4$ & $3 \cdot 00$ & $-8 \cdot 10$ & $-5 \cdot 49$ & $2 \cdot 98$ & $3 \cdot 52$ & $2 \cdot 27$ \\
\hline HD 168476 & $\mathrm{eHe}$ & 090099 & $182314 \cdot 66$ & $-563744 \cdot 1$ & $-0 \cdot 65$ & $0 \cdot 04$ & $-9 \cdot 08$ & $1 \cdot 30$ & $1 \cdot 22$ & $0 \cdot 87$ \\
\hline HD 173409 & $\mathrm{HdC}$ & 092115 & $184626 \cdot 63$ & $-312032 \cdot 1$ & $1 \cdot 95$ & $-0 \cdot 05$ & $-4 \cdot 45$ & $1 \cdot 57$ & $1 \cdot 97$ & $1 \cdot 19$ \\
\hline V CrA & $\mathrm{RCB}$ & 092207 & $184732 \cdot 31$ & $-380932 \cdot 3$ & $1 \cdot 18$ & $-6 \cdot 03$ & $-4 \cdot 83$ & $2 \cdot 77$ & $4 \cdot 12$ & $2 \cdot 65$ \\
\hline HD 175893 & $\mathrm{HdC}$ & 093181 & $1858 \quad 47 \cdot 29$ & $-293018 \cdot 0$ & $1 \cdot 38$ & $2 \cdot 26$ & $-3 \cdot 36$ & $1 \cdot 42$ & $1 \cdot 71$ & $1 \cdot 05$ \\
\hline SV Sge & $\mathrm{RCB}$ & 093987 & $190811 \cdot 78$ & $+173741 \cdot 2$ & 0.52 & $0 \cdot 82$ & $6 \cdot 65$ & $3 \cdot 56$ & $3 \cdot 05$ & $2 \cdot 92$ \\
\hline RY Sgr & $\mathrm{RCB}$ & 094730 & $191632 \cdot 76$ & $-333120 \cdot 3$ & $0 \cdot 36$ & $10 \cdot 33$ & $-0 \cdot 41$ & $1 \cdot 12$ & $1 \cdot 15$ & $0 \cdot 62$ \\
\hline HD 182040 & $\mathrm{HdC}$ & 095289 & $192310 \cdot 07$ & $-104211 \cdot 6$ & $0 \cdot 00$ & $7 \cdot 11$ & $1 \cdot 72$ & $0 \cdot 98$ & $0 \cdot 80$ & 0.50 \\
\hline V482 Cyg & $\mathrm{RCB}$ & 098411 & $195942 \cdot 58$ & $+335928 \cdot 0$ & $-8 \cdot 96$ & $-4 \cdot 84$ & $-12 \cdot 21$ & $3 \cdot 81$ & $3 \cdot 06$ & $4 \cdot 25$ \\
\hline $\mathrm{U}$ Aqr & $\mathrm{RCB}$ & 108876 & $2203 \quad 19 \cdot 70$ & $-163735 \cdot 3$ & $3 \cdot 58$ & $3 \cdot 03$ & $-2 \cdot 53$ & $2 \cdot 55$ & $3 \cdot 19$ & $1 \cdot 40$ \\
\hline
\end{tabular}

at maximum light ranging from $M_{\mathrm{bol}} \approx-4$ to -5 , with some probable scatter in $M_{\text {bol }}$ for stars of similar spectral type.

Distances to the Galactic $\mathrm{HdC}$ stars would usefully contribute to a number of outstanding issues concerning these types of objects, e.g. whether the different metallicity environments of the LMC and the Galaxy result in different luminosities, support the possibility of a period-luminosity relationship as suggested in the LMC RCB stars, in conjunction with atmospheric models provide intrinsic colours and values for circumstellar reddening due to the presence of dust, and provide a direct measure of the extent of large circumstellar features known to surround some RCB stars.

\section{Hipparcos Observations}

The Hipparcos satellite was launched by the European Space Agency during 1989 August, with scientific observations made between 1989 November and 1993 March. More than $10^{5}$ objects were observed for the Hipparcos Catalogue (ESA 1997), which has a mean astrometric precision of $\sim 1$ milliarcsec (mas). Table 1 lists raw parallaxes and proper motions obtained for HdC stars observed as part of the Hipparcos catalogue (the catalogue reference number for each star is the HIP number in Table 1).

\section{Discussion}

Of the $21 \mathrm{HdC}$ stars observed by Hipparcos, 20 have either null or negative parallaxes ( 7 stars) or less than $1.5 \sigma$ positive parallaxes (13 stars). None of these 13 positive parallaxes are significant. Indeed, if bias effects such as the Lutz-Kelker statistical bias correction (Lutz \& Kelker 1973; Koen 1992) are taken into account, these very weak positive values are most likely overestimates of the true parallax for each star.

The RCB star Y Mus is apparently detected at $\pi=8 \cdot 02 \pm 1 \cdot 93$ mas, i.e. a $4 \cdot 2 \sigma$ detection. Even a detection at this level is marginal for individual usefulness according to Koen (1992). However, even if the parallax was correct, the result is implausible for a RCB star. This parallax measurement corresponds to a distance of $126 \pm 30$ pc. At $m_{V} \approx 9 \cdot 3$ and $(B-$ $V)_{\mathrm{o}} \approx 0.6$ (Lawson et al. 1990 ; see Table 21 ), this would place the star at $M_{V} \approx 3 \cdot 8$ and therefore near the main sequence. Y Mus is spectroscopically similar to Galactic RCB stars such as RY Sgr and $\mathrm{R} \mathrm{CrB}$, and the LMC RCB stars W Men and HV 12842. These are all low-gravity stars, with $\log g \approx$ 0-1, which must imply an $M_{V}$ of at least -3 or -4 .

There is little likelihood that $\mathrm{Y}$ Mus has been misidentified with another star in the surrounding field. The star has no near neighbours of similar magnitude and the Hipparcos coordinate is accurate. The star was noted by ESA to be double or else affected by surrounding nebulosity (van Leeuwen, private communication). Either effect would render the parallax unreliable. Binarity would be surprising; none of the RCB stars or related objects are known to be in binary systems, which may be an indicator of their previous evolution. However, a number of RCB stars are known or suspected to be surrounded by extensive nebula (e.g. UW Cen; see Pollacco et al. 1991). But if either binarity or nebulosity is the correct explanation, Y Mus is unique in the class in showing such a highly distorted parallax.

We have attempted a mean parallax analysis for the 21 stars and derive $\overline{M_{V}} \sim-3$. The mean result is approximate to the expected value but is subject 
Table 2. Galactic distances, radial and space velocities, and theoretical parallaxes for cool hydrogen-deficient carbon stars

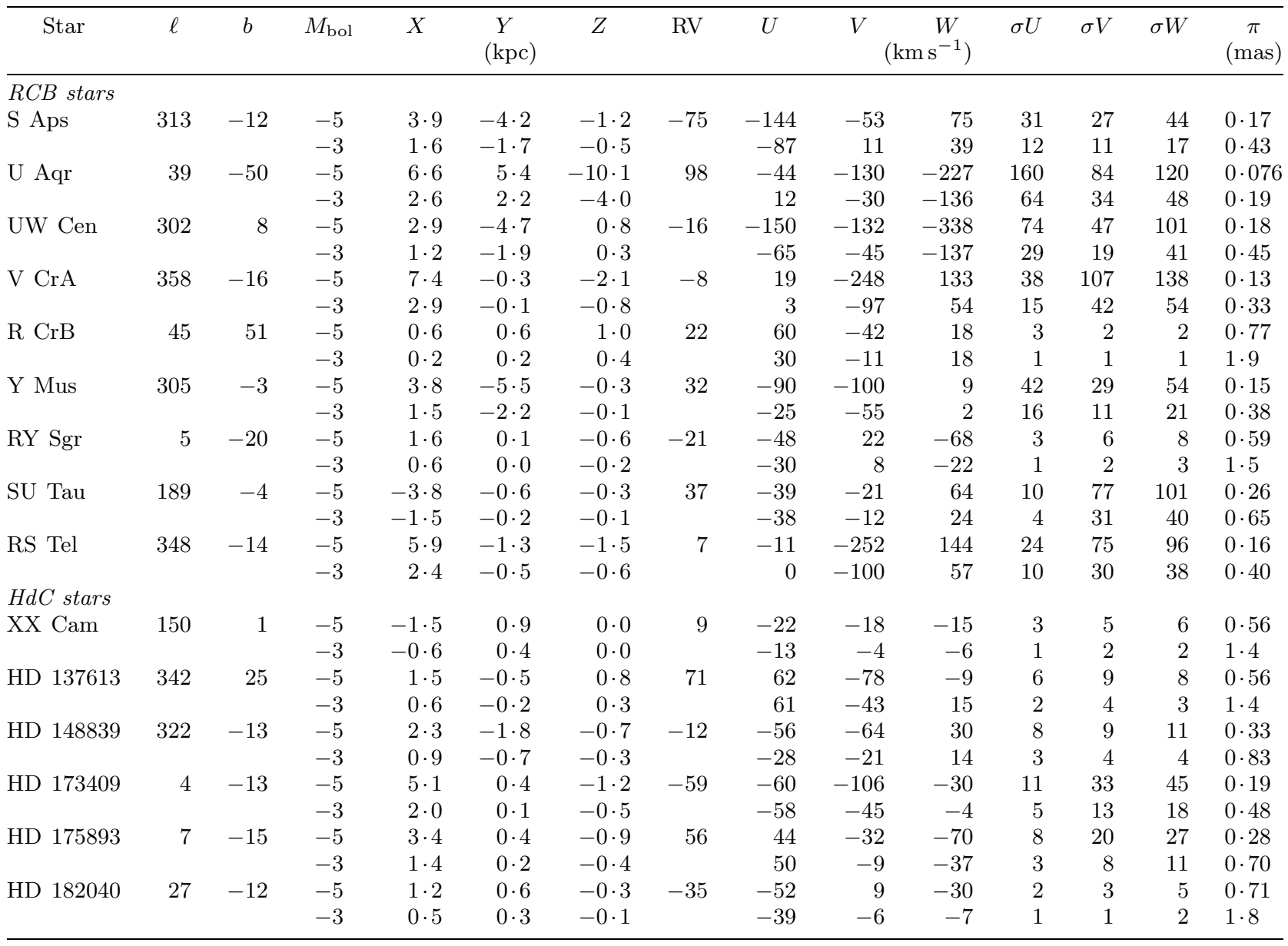

to large error and therefore must be interpreted with caution, e.g. the sample size is small, the mean parallax is small $(\bar{\pi}=0.65$ mas $)$, and there is uncertainty about the range of $M_{V}$ in these types of stars.

Table 2 lists cool HdC stars observed by Hipparcos for which we have reliable estimates of $m_{\text {bol }}$ (Lawson et al. 1990). In addition to their Galactic coordinates $(\ell, b)$ and heliocentric radial velocity (RV), we list Galactic distances $(X Y Z)$ with respect to the Sun and space velocities ( $U V W^{1}$, with $1 \sigma$ uncertainties) derived from the Hipparcos proper motions. The radial velocities for the $\mathrm{RCB} / \mathrm{HdC}$ stars were mainly sourced from Lawson \& Cottrell (1997), with the exception of SU Tau (Drilling \& Hill 1986) and XX Cam (Herbig, unpublished). A $0.5 \mathrm{~km} \mathrm{~s}^{-1}$ uncertainty $(1 \sigma)$ in the radial velocity has been assumed for each star, which also contributes to the space motion uncertainty. For each star the distances and space velocities were calculated assuming either $M_{\mathrm{bol}}=-5$ or -3 , which should encompass the likely range of luminosity for these stars. We also derive a theoretical parallax $\pi$ for each star at each luminosity.

\footnotetext{
${ }^{1} U$ is positive towards the Galactic centre, $V$ is positive in the direction of Galactic rotation and $W$ is positive towards the north Galactic pole.
}

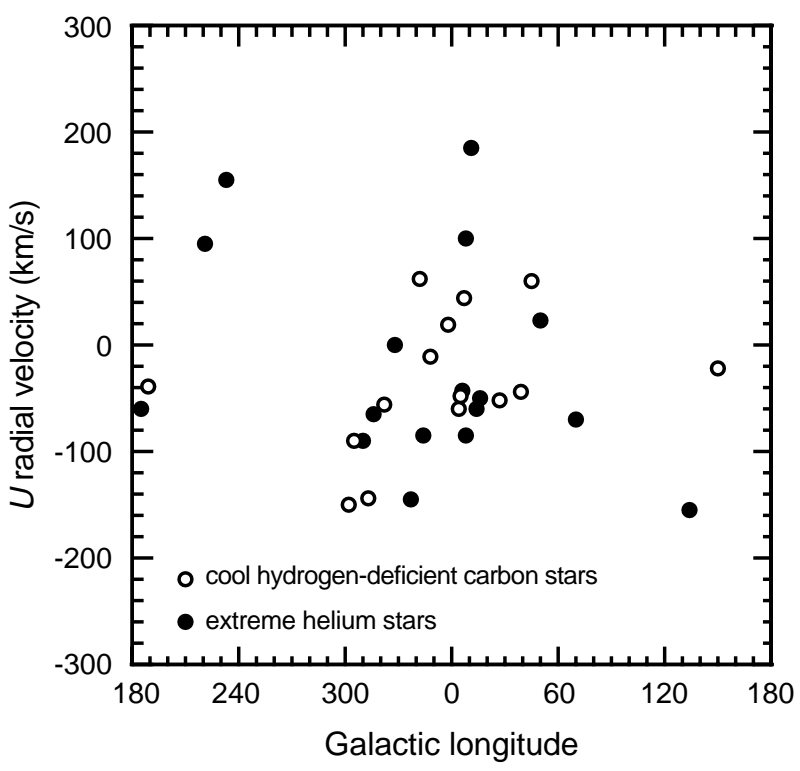

Figure 1-The $U$ radial velocity (positive towards the Galactic centre) versus Galactic longitude for cool hydrogen-deficient carbon stars (open circles) and extreme helium stars (filled circles; data from Drilling 1986). The velocity and spatial distribution of these types of stars is discussed in the text.

We have not produced $X Y Z, U V W$ values for the four eHe stars listed in Table 1 due to the large variation in $M_{V}$ across the eHe-star sequence 
(e.g. Drilling 1986; see Figure 1) and the large and uncertain bolometric correction required for these high-temperature stars.

Some of the results in Table 2 are similar to those obtained previously, but the Hipparcos-derived $U V W$ velocities are new. Drilling (1986) reported the distribution of some of the cooler $\mathrm{RCB} / \mathrm{HdC}$ stars and most of the known eHe stars in the $\ell-b$ plane and the $R V-\ell$ plane; both planes showed a distribution indicative of a bulge population. Lawson et al. (1990) and Lawson \& Cottrell (1990) showed a larger sample of cool $\mathrm{HdC}$ stars in the $X-Y$ and $X-Z$ planes, respectively, assuming $M_{\mathrm{bol}}=-5$. These show a known population skewed between $\ell=300$ and $360^{\circ}$, and avoiding much of the obscuration of the Galactic disk.

Assuming $M_{\mathrm{bol}}=-5$, the $U$-velocity dispersion of the $\mathrm{RCB} / \mathrm{HdC}$ stars listed in Table 2 is similar to that reported for the eHe stars by Drilling (1986). Figure 1 shows these stars in the $U$-velocity/Galactic longitude plane. Considering only those stars that lie within $60^{\circ}$ of the Galactic centre, the $U$-velocity dispersion of the $\mathrm{RCB} / \mathrm{HdC}$ stars is $65 \mathrm{~km} \mathrm{~s}^{-1}(1 \sigma)$, compared to $80 \mathrm{~km} \mathrm{~s}^{-1}(1 \sigma)$ for the eHe stars. The small difference in the velocity dispersion may be accounted for by the eHe star sample in Figure 1 being a largely complete representation of known eHe stars, whereas only the brightest $\mathrm{RCB} / \mathrm{HdC}$ stars are plotted, but it may simply be a function of the small sample sizes. The velocity and spatial distribution of both these types of stars is similar to that of other predominantly bulge populations, e.g. compact planetary nebulae (Drilling 1986).

With the exception of $\mathrm{U}$ Aqr and UW Cen, the $W$-velocity for the $\mathrm{RCB} / \mathrm{HdC}$ stars shows a dispersion $\left(60 \mathrm{~km} \mathrm{~s}^{-1} ; 1 \sigma\right)$ similar to that of the $U$ velocity. U Aqr is a halo RCB star, with a $Z$-distance of $-10 \mathrm{kpc}$. The high $W$-velocity of $-227 \mathrm{~km} \mathrm{~s}^{-1}$ is not unusual for a halo object. UW Cen is only 800 pc above the Galactic plane, unexceptional for the $\mathrm{RCB} / \mathrm{HdC}$ stars listed in Table 2, yet has a $W$-velocity of $-338 \mathrm{~km} \mathrm{~s}^{-1}$. UW Cen may be another halo RCB star, seen transitting the Galactic plane. However, this velocity does have large uncertainty and the conclusion is sensitive to the adopted absolute magnitude.

The theoretical parallaxes for R CrB, RY Sgr, XX Cam, HD 137613 and HD 182040 should have been detected by Hipparcos, even if with considerable uncertainty, if these stars were $M_{\mathrm{bol}}=-3$. Yet none of these stars returned a statistically significant parallax.
Thus we must await the next generation of parallax/proper motion engines to reliably characterise the distances, proper motions and luminosities of these types of stars. At least the Hipparcos measurements support the results obtained for the LMC hydrogen-deficient stars; the Galactic $\mathrm{RCB} / \mathrm{HdC}$ stars must also be high-luminosity objects.

\section{Acknowledgments}

We would like to thank the Hipparcos team of scientists and engineers who, over the last two decades, have worked to obtain significant advances in the determination of precision parallaxes and proper motions.

We also thank Geoff Clayton, Louisiana State University, for discussions about the new RCB stars discovered in the MACHO project LMC variable star inventory, and Floor van Leeuwen, RGO, for comments on the unusual parallax for Y Muscae.

The space velocities were obtained using software developed by Jovan Skuljan in the Department of Physics \& Astronomy at the University of Canterbury.

Alcock, C., et al. 1996, ApJ, 470, 583

Clayton, G. C. 1996, PASP, 108, 225

Clayton, G. C. 1998, in ISO's View on Stellar Evolution, ed. R. Waters, in press

Cottrell, P. L. 1996, in Hydrogen Deficient Stars, ed. C. S. Jeffery \& U. Heber, PASP Conf. Series, Vol. 96 (San Francisco: ASP), p. 13

Cottrell, P. L., \& Lambert, D. L. 1982, ApJ, 441, 400

Drilling, J. S. 1986, in Hydrogen Deficient Stars and Related Objects, ed. K. Hunger et al. (Dordrecht: Reidel), p. 9

Drilling, J. S., \& Hill, P. W. 1986, in Hydrogen Deficient Stars and Related Objects, ed. K. Hunger et al. (Dordrecht: Reidel), p. 499

ESA 1997, in The Hipparcos Catalogue, ESA SP-1200

Feast, M. W., \& Catchpole, R. M. 1997, MNRAS, 286, L1

Glass, I. S., Lawson, W. A., \& Laney, C. D. 1994, MNRAS, 270,347

Koen, C. 1992, MNRAS, 256, 65

Lambert, D. L., \& Rao, N. K. 1994, JA\&A, 15, 47

Lawson, W. A., \& Cottrell, P. L. 1990, in Confrontation between Stellar Pulsation and Evolution, ed. C. Cacciari \& G. Clementini PASP Conf. Series, Vol. 11 (San Francisco: ASP), p. 566

Lawson, W. A., \& Cottrell, P. L. 1997, MNRAS, 285, 266

Lawson, W. A., Cottrell, P. L., Kilmartin, P. M., \& Gilmore, A. C. 1990, MNRAS, 247, 91

Lutz, T. E., \& Kelker, D. H. 1973, PASP, 85, 573

Pollacco, D. L., Hill, P. W., Houziaux, L., \& Manfroid, J. 1991, MNRAS, 248, $1 \mathrm{P}$

Pollard, K. R., Cottrell, P. L., \& Lawson, W. A. 1994, MNRAS, 268, 544

Walker, H. J. 1986, in Hydrogen Deficient Stars and Related Objects, ed. K. Hunger et al. (Dordrecht: Reidel), p. 409 